\title{
Constraining (pseudo)Conformal Universe and anisotropic infla- tion with Planck data
}

\author{
Grigory I. Rubtsov ${ }^{1, \star}$ and Sabir R. Ramazanov ${ }^{2, \star \star}$ \\ ${ }^{1}$ Institute for Nuclear Research of the Russian Academy of Sciences, 60th October Anniversary st. 7a, \\ Moscow 117312, Russia \\ ${ }^{2}$ Gran Sasso Science Institute (INFN), viale Francesco Crispi 7, I-67100 L'Aquila, Italy
}

\begin{abstract}
We review the constraints on the (pseudo)conformal Universe and inflation from the non-observation of statistical anisotropy in the Planck'2013 maps provided at the frequencies $143 \mathrm{GHz}$ and $217 \mathrm{GHz}$, as well as their cross-correlation.
\end{abstract}

Statistical isotropy (SI) is one of the cornerstones of the modern cosmology. Deviations from this property - if observed in the cosmic microwave background (CMB) or large scale structure surveys would set a problem for most of the existing inflationary scenarios. Indeed, according to the Wald's non-hair conjecture, the Universe undergoes a rapid isotropization, what precludes the generation of any subtantial statistical anisotropy (SA). While some counterexamples to the Wald's conjecture are present on the market [1,2], they either involve ghost instabilities [3] or deal with the fine tuning issues [4]. These problems are absent in some alternatives to inflation, e.g., (pseudo)conformal Universe [5-7], which includes the conformal rolling scenario [5] and the Galilean genesis [6] as the particular realizations.

Statistical anisotropy from (pseudo)Confomal Universe. In this picture, the space-time geometry is effectively Minkowskian at very early times. The state of the Universe is invariant under the transformations of the conformal symmetry group $S O(4 \mid 2)$, spontaneously broken down the de Sitter subgroup $S O(4 \mid 1)$. The zero-weight conformal field present in the Universe evolves in the symmetry breaking background and its perturbations acquire the Harrison-Zel'dovich spectrum [5-7]. These field perturbations get reprocessed into adiabatic perturbations at much later epoch. The source of non-trivial phenomenology in this setup is the interaction between zero-weight field perturbations and the Goldstone field associated with the symmetry breaking pattern [8-11]. In particular, sufficiently long/short wavelength modes of the Goldstone field give rise to SA [8, 10]/non-Gaussianity [9].

In the conformal rolling scenario and the Galilean genesis, the symmetry breaking pattern $S O(4 \mid 2) \rightarrow S O(4 \mid 1)$ is achieved by introducing the field $\rho$ characterized by the conformal weight $\Delta=1$. The homogeneous background for the field $\rho$ is fixed by the dilatation invariance,

$$
\rho=\frac{1}{h\left(t_{*}-t\right)} .
$$

The constant $h$ here is the most important parameter of the conformal rolling scenario and Galilean genesis, as it governs the non-trivial phenomenology, including SA; $t_{*}$ is the constant of integration,

$\star$ e-mail: grisha@ms2.inr.ac.ru

$\star \star$ e-mail: sabir.ramazanov@gssi.infn.it 
which has the meaning of the end-of-roll time. The field $\theta$ with the conformal weight $\Delta=0$ evolves in the background (1) and acquires flat power spectrum at the times close to $t_{*}$. On the other hand, long ranged perturbations above the background (1) provoke the small distortion of the power spectrum manifesting as SA.

In the anisotropic Universe, the power spectrum allows for the following generic parametrization,

$$
\mathcal{P}_{\zeta}(\mathbf{k}) \propto\left(1+\sum_{L M} q_{L M}(k) Y_{L M}(\hat{\mathbf{k}})\right) .
$$

Here $Y_{L M}(\hat{\mathbf{k}})$ are the spherical harmonics, $\hat{\mathbf{k}}$ is the unit vector associated with the directions of the cosmological momentum $\mathbf{k}$, i.e., $\hat{\mathbf{k}}=\mathbf{k} / k$, and $q_{L M}(k)$ are the coefficients parametrizing SA.

Though the symmetry breaking pattern $S O(4 \mid 2) \rightarrow S O(4 \mid 1)$ uniquely fixes the shape of SA generated by the end of the roll, the subsequent evolution of the field $\theta$ may substantially alter its form. Baring fine-tuning, there are two interesting cases. First, the field $\theta$, frozen at the end of the roll, remains so until the beginning of the hot Big Bang. In this case, the form of SA is fully determined by the symmetry considerations. Another set of predictions takes place, if the field $\theta$ follows a long intermediate evolution in the Minkowski background after the end of the roll. These two options are referred to as the sub-scenarios A and B, respectively. The associated predictions for SA are summarized below.

Sub-scenario A. In that case, the directional dependence is of the quadrupolar form [8, 11],

$$
q_{2 M}=\frac{H_{0}}{k} q_{2 M}^{\prime}+q_{2 M}^{\prime \prime}
$$

Here $q_{2 M}^{\prime}$ 's and $q_{2 M}^{\prime \prime}$ 's encode contributions to SA appearing in the linear and quadratic orders in the parameter $h$, respectively. Note that the leading order (LO) contribution is characterized by the amplitude decreasing with the wavenumber. Coefficients $q_{2 M}^{\prime}$ obey the Gaussian statistics with the zero mean and variance

$$
\left\langle q_{2 M}^{\prime} q_{2 M^{\prime}}^{\prime *}\right\rangle=\frac{\pi h^{2}}{25} \delta_{M M^{\prime}} .
$$

The sub-leading order (SLO) contribution is of the axisymmetric form. Namely,

$$
q_{2 M}^{\prime \prime}=-\frac{4 \pi v^{2}}{5} Y_{2 M}^{*}(\hat{\mathbf{v}})
$$

where $\hat{\mathbf{v}}=\mathbf{v} / v$. The components of the vector $\mathbf{v}$ are the Gaussian quantities characterized by the zero means and the dispersions,

$$
\left\langle v_{i}^{2}\right\rangle=\frac{3 h^{2}}{8 \pi} \ln \frac{H_{0}}{\Lambda} .
$$

Here the present Hubble rate $H_{0}$ and the scale $\Lambda$ play a role of the ultraviolet and the infrared cutoffs for the long ranged modes of the perturbations of the field $\rho$, which serve as the source of SA.

Sub-scenario B. The structure of SA is particularly rich in that case [10]. Namely, all the coefficients $q_{L M}$ with even $L$ are non-zero in (2). They are the Gaussian quantities with zero means and variances given by

$$
\left\langle q_{L M} q_{L^{\prime} M^{\prime}}^{*}\right\rangle=\frac{3 h^{2}}{\pi(L-1)(L+2)} \delta_{L L^{\prime}} \delta_{M M^{\prime}} .
$$

Inflation. As it was pointed out in the very beginning, SI is the outcome in the bulk of inflationary scenarios. There are some exceptions, however. The ghost free scenario of inflation capable of generating non-vanishing SA has been put forward in Ref. [2]. There one deals with the Maxwellian field 

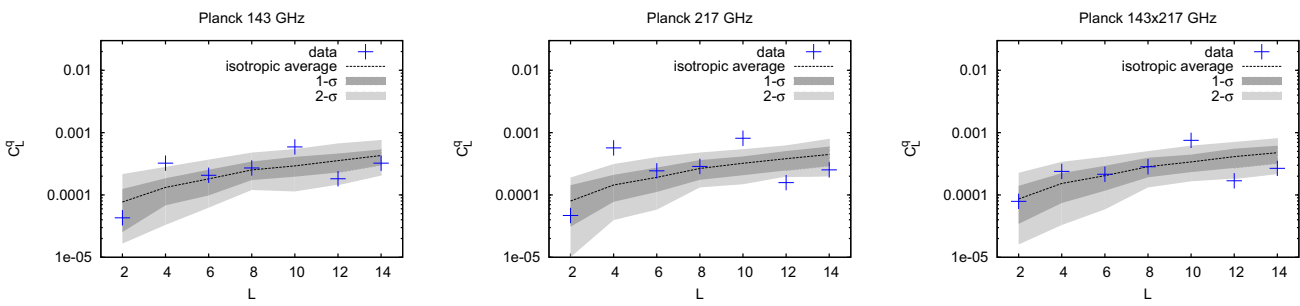

Figure 1. Coefficients $C_{L}^{q}$ given by Eq. (11) reconstructed from the Planck data [18]. The scale invariance of the parameters $q_{L M}$ in Eq. (2), i.e., $q_{L M}(k)=q_{L M} .68 \%$ and 95\% C.L. intervals are overlaid with dark grey and light grey, respectively.

non-minimally coupled to the inflaton field. This coupling allows for the prolonged anisotropic evolution of the inflationary Universe, with the preferred direction stretched along the the electric vector $\mathbf{E}$. Inflaton perturbations evolving in this anisotropic background, acquire the direction-dependent power spectrum. Modulo the overall factor and the replacement $\mathbf{v} \leftrightarrow \mathbf{E}$, the shape of the resultant SA is exactly the same as in the SLO of the pseudo-conformal Universe. Choosing the $z$-axis of the cosmic reference frame along the preferred direction (the direction of the vector $\mathbf{E}$ ), one gets for the power spectrum,

$$
\mathcal{P}_{\zeta} \propto\left(1+g_{*} \cos ^{2} \theta\right)
$$

This can be rewritten in the generic form (2), with the only non-zero coeffients $q_{2 M}$ related to the amplitude $g_{*}$ by

$$
q_{2 M}=\frac{8 \pi g_{*}}{15} Y_{2 M}^{*}(\hat{\mathbf{E}}) .
$$

When treating anisotropic inflationary scenarios, one typically constrains the amplitude $g_{*}$. This constraint then can be easily converted into the bounds on the intrinsic model parameters.

In the sitiuation, when there are several Maxwellian fields non-minimally coupled to the inflaton [12], the form of the resultant SA is still of the quadrupolar form, but no more possesses the axial symmetry [13]. This case is captured by the following generalization of Eq. (8),

$$
\mathcal{P}_{\zeta} \propto\left(1+\sqrt{\frac{16 \pi}{45}} g_{*} Y_{20}(\vartheta, \varphi) \cos \chi-\sqrt{\frac{8 \pi}{45}} g_{*}\left(Y_{2,1}(\vartheta, \varphi)-Y_{2,-1}(\vartheta, \varphi)\right) \sin \chi\right) .
$$

Here $\chi$ is the additional parameter. It has the meaning of the angle between two multipole vectors describing the general quadrupole. When writing Eq. (10), the particular choice of the reference frame is implied: the $z$-axis is aligned with one of the multipole vectors, while the second multipole vector lies in the $O x z$ plane.

Constraints. In our paper [18], we used the Planck CMB temperature maps corresponding to the first 15.5 months of observation at the frequencies $143 \mathrm{GHz}$ and $217 \mathrm{GHz}$ [19, 20]. To remove the contamination of the galactic light and point sources we applied the High Frequency Instrument (HFI) power spectrum mask [20,21], which leaves $43 \%$ of the sky unmasked. Averaging over statistically isotropic realizations was performed using 100 Planck simulated multi-frequency CMB maps coadded with the corresponding noise maps and the foreground maps [20, 22]. These maps incorporate the effects of beam asymmetries and complex scanning strategy [23], which are proved to be crucial for the SI studies [17, 22, 24]. The operations with the maps have been performed with the HEALPix and healpy packages [25]. 

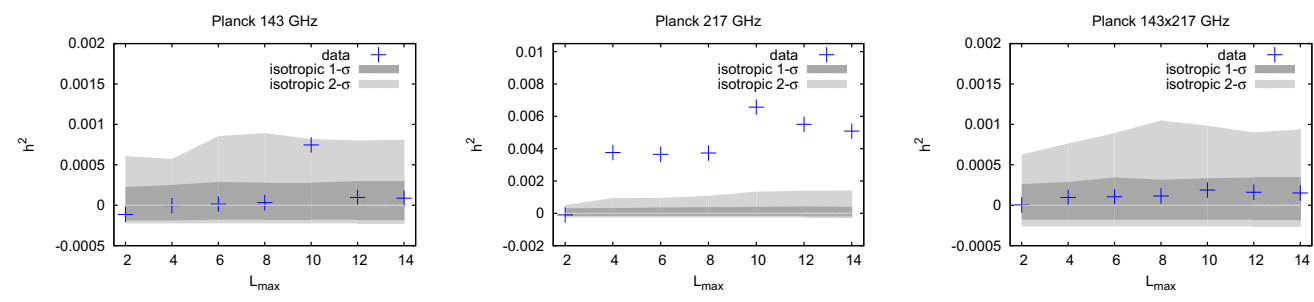

Figure 2. Parameter $h^{2}$ of the (pseudo)conformal Universe with long intermediate stage estimated from the Planck data. $68 \%$ and $95 \%$ C.L. intervals are overlaid with dark grey and light grey, respectively.

We start with constraining sub-scenario A of the (pseudo)conformal Universe. To estimate the parameter $h^{2}$, we use the statistics given by the coefficients $C_{L}^{q}$,

$$
C_{L}^{q}=\frac{1}{2 L+1} \sum_{M}\left|q_{L M}\right|^{2},
$$

where we set $L=2$. To reconstruct the parameters $q_{2 M}$, we make use of the quadratic maximum likelihood techniques [14]. The latter has proved to be an efficient tool for searches of SA in the WMAP and Planck data [15-18]. Results are plotted in Fig. 1. Clearly, Planck'2013 data are consistent with the hypothesis of SI. The small excess in the $217 \mathrm{GHz}$ frequency band present at the multipole number $L=4$ vanishes upon cross-correlating with the $143 \mathrm{GHz}$ frequency band. Hence, it should be considered as the noise/systematical effect rather than the signal of the primordial SA.

For brevity, we discuss only the constraining procedure for the case of the SLO contribution to SA. This is in fact the most relevant contribution, as the LO effect is suppressed by the wavenumber $k$. First, we make use of Eq. (6) to generate a large number of Gaussian vectors $\mathbf{v}$ at the initial step. Next we use Eq. (5) to convert vectors $\mathbf{v}$ into the sets of coefficients $q_{2 M}^{\prime \prime}$. For each set, we generate one anisotropic map, and reconstruct the coefficient $C_{2}^{q}$. We demand that no more than $95 \%$ of the $C_{2}^{q}$ values from simulated maps should exceed the value of the coefficient $C_{2}^{q}$ obtained from the data. The final constraints originating from the LO and SLO contributions to SA are presented in Table 1. As it is expected, the SLO effect yields a stronger constraint than the LO suppressed by the wavenumber $k[15,16]$.

\begin{tabular}{|c|c|c|c|c|}
\hline Model/band & $143 \mathrm{GHz}$ & $217 \mathrm{GHz}$ & $143 \times 217 \mathrm{GHz}$ & WMAP9, V \\
\hline Sub-scenario A (LO) & $h^{2}<8.8$ & $h^{2}<8.0$ & $h^{2}<3.0$ & $h^{2}<11$ \\
\hline Sub-scenario A (NLO) & $h^{2} \ln \frac{H_{0}}{\Lambda}<0.34$ & $h^{2} \ln \frac{H_{0}<0.30}{\Lambda}$ & $h^{2} \ln \frac{H_{0}}{\Lambda}<0.52$ & $h^{2} \ln \frac{H_{0}}{\Lambda}<1.2$ \\
\hline Sub-scenario B & $h^{2}<0.0011$ & $h^{2}<0.0090$ & $h^{2}<0.0013$ & $h^{2}<0.006$ \\
\hline Inflation & $\left|g_{*}\right|<0.020$ & $\left|g_{*}\right|<0.020$ & $\left|g_{*}\right|<0.026$ & $\left|g_{*}\right|<0.046$ \\
\hline
\end{tabular}

Table 1. Planck 95\% C.L. constraints on the parameter $h^{2}$ of (pseudo)conformal Universe and on the amplitude of the axisymmetric quadrupole anisotropy $g_{*}$ [18]. For the comparison purposes, we also present the analogous constraints obtained from the $V$ band of the WMAP9 data [16].

To constrain the parameter $h^{2}$ in the sub-scenario $\mathrm{B}$, the $C_{2}^{q}$ statistics is not enough, since all the coeffcients $q_{L M}$ with even $L$ are non-zero now. The relevant statistics can be found in Ref. [15], and has a generic form $h^{2}=F\left(C_{2}^{q}, C_{4}^{q}, \ldots\right)$. The results of implementing this estimator for the Planck data 

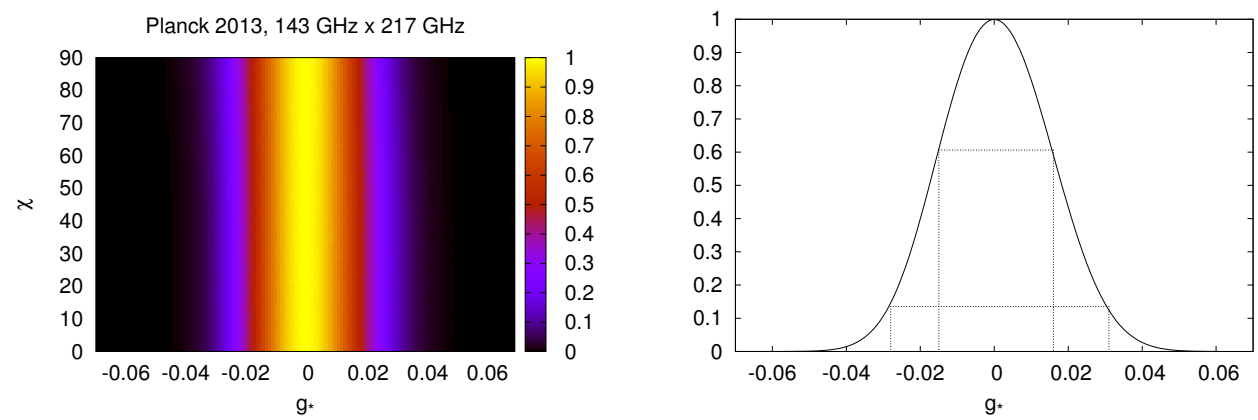

Figure 3. Planck Likelihood plotted as a function of the parameters $g_{*}$ and $\chi$, whcih describe the general quadrupolar SA (left plot), see Eq. (10). We see that it is independent on the angle $\chi$. The truncated likelihood distribution shown in the right plot implies the 68\% limits on the amplitude $g_{*}=0.001 \pm 0.015$, essentially the same as the ones derived in Ref. [17].

are shown in Fig. 2. Values of the estimators are plotted for seven ranges of the multipoles starting from the quadrupole $L=2$ and extending up to $L_{\max }=2, \ldots, 14$. The results for the frequency band $217 \mathrm{GHz}$ clearly exhibit SA, which is again due to the enhancement at $L=4$, see Fig. 1 . The effect vanishes upon cross-correlating the 143 and $217 \mathrm{GHz}$ frequency bands. The rest of the procedure is the same as in the sub-scenario A (see also the papers $[15,16,18]$ ), but now we use Eq. (7) to generate the sets of coefficients $\left\{q_{L M}\right\}$ for some particular value of the parameter $h^{2}$. Final constraints are presented in Table 1.

To bound the amplitude $g_{*}$ relevant for some inflationary scenarios, one folllows the simplified version of the procedure used to constrain the sub-scenario A. Namely, for each fixed value of $g_{*}$, we readily construct the set of parameters $q_{2 M}$ using Eq. (9), and then generate one anisotropic map for each set. The final bounds are given in Table 1. We finish with discussing the general quadrupolar SA arising in inflation equipped with multiple Maxwellian fields. Our preliminary results are shown in Fig. 3. As it is clearly seen, the Planck'2013 does not allow to distinguish between the cases of the quadrupolar SA with axial symmetry and without. Hence, no constraints for the parameter $\chi$ are set at this level, while the bounds on the amplitude $g_{*}$ remain intact.

We conclude that SA is a significant signature in the sub-scenario B of the (pseudo)conformal Universe. The constraints obtained from the Planck'2013 data demonstrate a factor 5 improvement compared to those derived from WMAP9 maps. On the other hand, SA is a relatively weak signature of the sub-scenario A, as is clearly seen from Table 1 . In that case, the advantage of using more sensitive data gives just a factor 2 improvement compared to the previous constraints. The Planck'2013 limits on the amplitude $g_{*}$ [18] are in a good agreement with the analogous ones [17] derived with the different constraining procedure. For the first time, we address the issue of the general quadrupole anisotropy produced by the inflationary scenarios with multiple vector fields. It appears, however, that the Planck'2013 data is not able to discriminate this case from the standard one. We plan to get back to this issue in the nearest future.

Acknowledgments. The work of GR is supported by the Russian Science Foundation Grant No. 1412-01430. 


\section{References}

[1] L. Ackerman, S. M. Carroll and M. B. Wise, Phys. Rev. D 75 (2007) 083502 [Erratum-ibid. D 80 (2009) 069901] [astro-ph/0701357].

[2] M. -a. Watanabe, S. Kanno and J. Soda, Phys. Rev. Lett. 102 (2009) 191302 [arXiv:0902.2833 [hep-th]].

[3] B. Himmetoglu, C. R. Contaldi and M. Peloso, Phys. Rev. D 79 (2009) 063517 [arXiv:0812.1231 [astro-ph]].

[4] N. Bartolo, S. Matarrese, M. Peloso and A. Ricciardone, Phys. Rev. D 87 (2013) 023504 [arXiv:1210.3257 [astro-ph.CO]].

[5] V. A. Rubakov, JCAP 0909 (2009) 030 [arXiv:0906.3693 [hep-th]].

[6] P. Creminelli, A. Nicolis and E. Trincherini, JCAP 1011 (2010) 021 [arXiv:1007.0027 [hep-th]].

[7] K. Hinterbichler and J. Khoury, JCAP 1204 (2012) 023 [arXiv:1106.1428 [hep-th]].

[8] M. Libanov and V. Rubakov, JCAP 1011 (2010) 045 [arXiv:1007.4949 [hep-th]].

[9] M. Libanov, S. Mironov and V. Rubakov, Phys. Rev. D 84 (2011) 083502 doi:10.1103/PhysRevD.84.083502 [arXiv:1105.6230 [astro-ph.CO]].

[10] M. Libanov, S. Ramazanov and V. Rubakov, JCAP 1106 (2011) 010 [arXiv:1102.1390 [hep-th]].

[11] P. Creminelli, A. Joyce, J. Khoury and M. Simonovic, JCAP 1304 (2013) 020 doi:10.1088/14757516/2013/04/020 [arXiv:1212.3329].

[12] M. Thorsrud, D. F. Mota and F. R. Urban, Phys. Lett. B 733 (2014) 140 doi:10.1016/j.physletb.2014.04.028 [arXiv:1311.3302 [astro-ph.CO]].

[13] M. Thorsrud, F. R. Urban and D. F. Mota, JCAP 1404 (2014) 010 doi:10.1088/14757516/2014/04/010 [arXiv:1312.7491 [astro-ph.CO]].

[14] D. Hanson and A. Lewis, Phys. Rev. D 80 (2009) 063004 [arXiv:0908.0963 [astro-ph.CO]].

[15] S. R. Ramazanov and G. I. Rubtsov, JCAP 1205 (2012) 033 [arXiv:1202.4357 [astro-ph.CO]].

[16] S. R. Ramazanov and G. Rubtsov, Phys. Rev. D 89 (2014) 043517 [arXiv:1311.3272 [astroph.CO]].

[17] J. Kim and E. Komatsu, Phys. Rev. D 88 (2013) 101301 [arXiv:1310.1605 [astro-ph.CO]].

[18] G. I. Rubtsov and S. R. Ramazanov, Phys. Rev. D 91 (2015) no.4, 043514 doi:10.1103/PhysRevD.91.043514 [arXiv:1406.7722 [astro-ph.CO]].

[19] P. A. R. Ade et al. [Planck Collaboration], Astron. Astrophys. 571, A1 (2014) doi:10.1051/00046361/201321529 [arXiv:1303.5062 [astro-ph.CO]].

[20] http://www.sciops.esa.int/wikiSI/planckpla/index.php

[21] P. A. R. Ade et al. [Planck Collaboration], Astron. Astrophys. 571, A15 (2014) [arXiv:1303.5075 [astro-ph.CO]].

[22] P. A. R. Ade et al. [Planck Collaboration], Astron. Astrophys. 571, A23 (2014) [arXiv:1303.5083 [astro-ph.CO]].

[23] S. Mitra, G. Rocha, K. M. Gorski, K. M. Huffenberger, H. K. Eriksen, M. A. J. Ashdown and C. R. Lawrence, Astrophys. J. Suppl. 193 (2011) 5 [arXiv:1005.1929 [astro-ph.CO]].

[24] D. Hanson, A. Lewis and A. Challinor, Phys. Rev. D 81 (2010) 103003 [arXiv:1003.0198 [astroph.CO]].

[25] K. M. Gorski, E. Hivon, A. J. Banday, B. D. Wandelt, F. K. Hansen, M. Reinecke and M. Bartelman, Astrophys. J. 622, 759 (2005) [astro-ph/0409513]. 